\title{
CARIES DENTAL E HIGIENE BUCAL EN ESCOLARES DE LA ZONA RURAL DEL MUNICIPIO DE JERICÓ, ANTIOQUIA, 2013
}

\author{
Wilder Javier Rojas-Gutiérrez, Odont. ${ }_{1}$, Anny M. Vivares-Builes, Odont. ${ }_{2}$, \\ Andrés A. Agudelo-Suárez ${ }^{*}$ Ph.D.
}

${ }_{1}$ Facultad de Odontología, Universidad de Antioquia, Colombia.

${ }_{2}$ Facultad de Odontología, Universidad de Antioquia y Fundación Universitaria Autónoma de las Américas, Colombia.

Recibido: 23 de septiembre del 2013. Aprobado: 21 de octubre del 2013.

*Autor de correspondencia: Andrés A. Agudelo-Suárez, Facultad de Odontología, Universidad de Antioquia, Medellín, Colombia, teléfono: (57) 42196772 , Calle 70 n. 52-21, correo electrónico: oleduga@gmail.com

Cómo citar este artículo: Rojas-Gutiérrez WJ, Vivares-Builes AM, Agudelo-Suárez AA. Caries dental e higiene bucal en escolares de la zona rural del municipio de Jericó, Antioquia, 2013. Rev Nac Odontol. 2013; 9(17): 27-34.

Resumen. Introducción: los objetivos de esta investigación fueron determinar la prevalencia y experiencia de caries dental en la población escolar rural del municipio de Jericó (Antioquia) y analizar las condiciones de higiene bucal de la población de estudio. Métodos: estudio descriptivo con la información de 534 historias clínicas de personas entre los 6 y 22 años, con dentición mixta o permanente del área de estudio. Se analizaron variables sociodemográficas, Índice de Higiene Oral Simplificado (IHOs), prevalencia y experiencia con caries dental en dentición permanente mediante el índice cop tradicional y modificado $\left(\operatorname{COPD}_{\mathrm{T}} \mathrm{y} \mathrm{COPD}_{\mathrm{M}}\right)$ y el Índice de Caries Significativo (sIC). Análisis uni y bivariado y cálculo de $\mathrm{Chi}^{2}$ para observar diferencias significativas entre las variables. Resultados: la prevalencia de caries dental en ambos sexos, incluyendo lesiones no cavitacionales, es del 39\%, y la experiencia con caries es del $47 \%$ en hombres y el $46 \%$ en mujeres. Teniendo en cuenta el sic, el promedio en hombres es de 2,70 cuando se calcula a partir del $\mathrm{COPD}_{\mathrm{T}}$ y del 4,36 para el caso del $\mathrm{COPD}_{\mathrm{M}}$. Estos indicadores son menores en mujeres (2,56 y 3,36, respectivamente). En cuanto al IHOs, el promedio es mucho menor en mujeres (1,25 IC95\% 1,18-1,32) y con diferencias estadísticamente significativas con respecto a los hombres $(\mathrm{p}<0,001)$. Se presentan diferencias en los indicadores de caries dental e higiene bucal, según escolaridad y edad. Conclusión: si bien los indicadores están de acuerdo con las metas internacionales, se encuentran algunos grupos poblacionales en situación de desigualdad.

Palabras clave: caries dental, escolares, higiene bucal, índice COPD, índice de caries significativo.

\section{Dental Caries and Oral Hygiene in School Children in the Rural Area of the Municipality of Jericó, Antioquia, 2013}

Abstract. Introduction: The objectives of this research were to determine the prevalence and experience of dental caries among school children in the rural area of the municipality of Jericó (Antioquia) and to analyze oral hygiene conditions in the study population. Methods: A descriptive study was carried out based on information from 534 clinical records of people between the ages of 6 and 22, with mixed and/or permanent dentitions located in the study area. We analyzed Socio-demographic, Simplified Oral Hygiene Index (IHos from the Spanish acronym), prevalence and experience of dental cavities in permanent dentition using the traditional and modified DMTF Index $\left(\mathrm{DMTF}_{\mathrm{T}}-\mathrm{DMTF}_{\mathrm{M}}\right)$ and the Significant Caries Index (sIC). Univariate and bivariate analyses were conducted and a Chi square test was performed to observe statistically significant differences between the variables. Results: the prevalence of dental caries including non-cavitated lesions is $39 \%$ among both genders; dental caries had been experienced by $47 \%$ of the men and $46 \%$ of the women. Taking into account the sIC, the mean is 2.70 when based on the DT$\mathrm{MF}_{\mathrm{T}}$ and 4.36 on the $\mathrm{DTMF}_{\mathrm{M}}$. These indicators are lower in women $(2.56$ and 3.36, respectively). The mean for the IHOs is lower among women
Cárie dental e higiene bucal em crianças da zona rural do município de Jericó, Antioquia, 2013

Resumo. Introdução: os objetivos desta pesquisa foram determinar a prevalência e experiência de cárie dental na população escolar rural do município de Jericó (Antioquia) e analisar as condições de higiene bucal da população de estudo. Métodos: estudo descritivo com a informação de 534 prontuários de pessoas entre 6 e 22 anos, com dentição mista e/ ou permanente da área de estudo. Analisaram-se variáveis sociodemográficas, Índice de Higiene Oral Simplificado (IHOS), prevalência e experiência com cárie dental em dentição permanente mediante o índice COP tradicional e modificado $\left(\mathrm{COPD}_{\mathrm{T}}\right.$ e $\left.\mathrm{COPD}_{\mathrm{M}}\right)$ e o Índice de Cárie Significativo (sIC). Análises uni e bivariada e cálculo de $\mathrm{Chi}^{2}$ para observar diferenças significativas entre as variáveis. Resultados: a prevalência de cárie dental em ambos os sexos, o que inclui lesões não cavitacionais, é de $39 \%$, e a experiência com cáries é de $47 \%$ em homens e $46 \%$ em mulheres. Ao considerar o sIC, a média em homens é de 2,70 quando se calcula a partir do $\mathrm{COPD}_{\mathrm{T}}$ e de 4,36 para o caso do $\mathrm{COPD}_{\mathrm{M}}$. Esses indicadores são menores nas mulheres (2,56 e 3,36, respectivamente). Quanto ao IHOs, a média é muito menor em mulheres ( 1,25 IC95\% 1,18-1,32) e com diferenças estatisticamente significativas com relação aos homens 
(1.25 95\%cI 1.18-1.32), with statistically significant differences compared to men $(\mathrm{p}<0.001)$. Differences in frequencies were observed in the dental caries and oral hygiene indicators according to age and level of education. Conclusion: Although the indicators are in accordance with international goals, inequalities were found among certain population groups.

Keywords: dental caries, scholars, oral hygiene, DMTF index, significant caries index.

\section{Introducción}

Los estudios epidemiológicos continúan situando al proceso de caries dental como una patología crónica altamente prevalente a nivel mundial, que afecta sin distinción a todos los grupos etarios y raciales. Se presentan diferencias en su distribución, teniendo en cuenta los fenómenos de polarización en los que algunas comunidades por sus condiciones sociales, económicas y demográficas presentan mayor prevalencia y experiencia con caries dental, asumiendo la mayor carga de la enfermedad, en términos económicos, psicosociales y biológicos, con un detrimento significativo de su calidad de vida [1-3].

Concretamente en Colombia, de acuerdo con los estudios nacionales de salud bucal, en las últimas tres décadas se ha presentado una disminución significativa en la severidad de la caries dental, pasando de un nivel muy alto a uno muy bajo, según la clasificación de la Organización Mundial de la Salud (oms) [2, 4-6]. No obstante, la prevalencia de caries continua siendo alta en la población adulta joven: es del $71 \%$ entre los $15 \mathrm{y}$ 19 años [6]. La línea de base de Antioquia (2011) reporta una prevalencia de caries del $68 \%$, incluyendo las lesiones de caries iniciales y cavitacionales [7].

En la actualidad existen nuevos conceptos en la clasificación, diagnóstico y tratamiento de la caries dental, gracias a una mejor comprensión de la etiología y la patogenia de esta enfermedad, lo que ha posibilitado desarrollar tratamientos menos invasivos y más preventivos, desde sus estadios iniciales, por lo que ha quedado la odontología con tendencia totalmente restauradora como una práctica del pasado $[8,9]$.

En el marco de estos nuevos conceptos de valoración del proceso de caries dental existen diferentes indicadores disponibles para su evaluación en grupos poblacionales específicos. Algunos de estos índices son considerados como simples en su aplicación, procesamiento y análisis, y otros como más complejos pero con aportes en términos de precisión diagnóstica y asociación con otros determinantes sociales. La selección de
( $\mathrm{p}<0,001)$. Apresentam-se diferenças nos indicadores de cárie dental e higiene bucal segundo a escolaridade e a idade. Conclusão: embora os indicadores estejam de acordo com as metas internacionais, constatamse alguns grupos populacionais em situação de desigualdade.

Palavras-chave: cárie dental, crianças em idade escolar, índice copD, índice de cárie significativo, higiene bucal.

los diferentes índices para monitorear el proceso de caries dental en una población va a estar ligada a la disponibilidad de recursos humano y técnico, y al interés por el establecimiento de un perfil epidemiológico en aras del logro de metas de salud, del fortalecimiento de los sistemas de información, de la posibilidad de generar un conocimiento más amplio del proceso de caries dental, de una evaluación del desempeño del sistema de salud y de los prestadores en determinada comunidad, permitiendo un seguimiento y una vigilancia epidemiológica como herramienta básica en la toma de decisiones y un planteamiento de estrategias enmarcadas en programas y proyectos de mayor impacto en la salud pública [9].

En Jericó, Antioquia (Colombia), mediante la Empresa Social del Estado (ESE) Hospital San Rafael se ha acrecentado el interés por conocer y marcar un precedente sobre la vigilancia epidemiológica en torno a la caries dental y su morbilidad, en especial en las áreas rurales del municipio, con miras a realizar planes de intervención para esta población. Por lo tanto, el presente estudio tiene como objetivos: 1) Determinar la prevalencia y experiencia con la caries dental en la población escolar rural de Jericó (Antioquia), y 2) Analizar las condiciones de higiene bucal de la población de estudio.

\section{Métodos}

Este es un estudio descriptivo transversal. Los datos fueron suministrados por la ESE Hospital San Rafael de Jericó para el 2013. La información fue recogida por medio de un levantamiento epidemiológico en el área rural conformada por 21 veredas, a cargo de un odontólogo estandarizado (quien además es investigador del proyecto y realizó los exámenes clínicos). Concretamente, en este estudio se utilizó la información de 534 historias clínicas odontológicas de personas 
entre los 6 y 22 años, con dentición mixta y permanente y una distribución más o menos similar entre hombres y mujeres. La selección de los participantes del estudio se realizó teniendo en cuenta la planeación institucional.

Se tomaron como variables de resultado: 1) Índice de higiene oral simplificado de Green y Vermillon (IHOs) [10]: este es un indicador que mide la presencia de placa blanda en dientes deciduos y permanentes. Se registra el promedio y va en un rango de 0 a 3,0 (a más promedio, más presencia de placa); 2) Con la información del IHos se obtuvo el nivel de higiene oral: bueno (0-1,29), regular, (1,30-3,09) y malo (>3,10); el Índice de dientes cariados, obturados y perdidos por caries (COPD) de Klein y Palmer [11], igualmente se tuvo en cuenta el COPD tradicional $\left(\mathrm{COPD}_{\mathrm{T}}\right)$ y el COPD modificado $\left(\mathrm{COPD}_{\mathrm{M}}\right)$; la modificación consistió en incluir en el registro las lesiones activas no cavitacionales [12]; 3) Índice de caries significativo (SiC) [13]: el análisis de la situación de la caries dental en muchos países ha mostrado que existe una distribución sesgada del copD. Por lo tanto, se incluye el SiC, que consiste en calcular el promedio COPD en el tercio más afectado de la población; para este caso se calcularon a partir del $\mathrm{COPD}_{\mathrm{T}}$ y el $\mathrm{COPD}_{\mathrm{M}}$, por medio de las fórmulas en hoja de cálculo disponibles en: http://www. mah.se/CAPP/Methods-and-Indices/for-Caries-prevalence/Significant-Caries-Index/Online-Calculation-of-DMFTdmft-and-SIC-Index-/; 4) Mediante el índice $\mathrm{COPD}_{\mathrm{T}} \mathrm{y}$ el $\mathrm{COPD}_{\mathrm{M}}$ se calcularon la prevalencia de caries dental (para todas las lesiones y las lesiones cavitacionales), lo mismo que la experiencia con caries dental. Como variables sociodemográficas se incluyeron: edad (6-9, 10-12, 13 y más) y grado escolar (primaria/ secundaria).

Se realizó un análisis uni- y bivariado: se calcularon promedios (desviación estándar-intervalos de confianza al 95\%) y frecuencias absolutas y relativas, tanto en forma general como por cada una de las variables sociodemográficas. Se realizaron Pruebas no paramétricas U de Mann Whitney y Kruskal Wallis y $\mathrm{Chi}^{2}$ para diferencia de proporciones, con el fin de observar la significación estadística entre las variables (teniendo en cuenta que el COP no sigue una distribución normal). Para el análisis de los datos se utilizaron los programas Excel para Windows, sPss 18.0 (IBM) y EPIDAT 3.1 (Dirección Xeral de Innovación e Xestión da Saúde Pública, Xunta de Galicia, España, Organización Panamericana de la Salud e Instituto Superior de Ciencias Médicas de La Habana).
Se manejaron los protocolos de examen odontológico, normas de bioseguridad y consentimiento informado, protección de la identidad y la veracidad de la información. La presente investigación cumple con la normativa internacional (Declaración de Helsinki) [14] y nacional (Resolución 008430 del Ministerio de Salud y Protección Social [antes, Ministerio de Salud]) [15] para investigación en salud, considerándose como un estudio de riesgo mínimo.

\section{Resultados}

En la tabla 1 se puede observar la distribución de las variables de la población de estudio y el índice COPD y sus diferentes componentes. En hombres existen gradientes con tendencia a un mayor promedio de dientes cariados, obturados y perdidos conforme aumenta la edad. Igual situación se presentó para los índices $\mathrm{COPD}_{\mathrm{T}}$ y $\operatorname{COPD}_{\mathrm{M}}$. Se encontraron diferencias estadísticamente significativas en la distribución porcentual de las variables ( $\mathrm{p}<0,001$ ), excepto para el caso de los dientes cariados no cavitacionales. En cuanto a las mujeres, los gradientes fueron menos evidentes al considerar los cariados no cavitacionales y los cariados en conjunto, aunque la tendencia de los indicadores aumenta con la edad, y se encontraron diferencias estadísticamente significativas $(p<0,01)$, excepto para el caso de los cariados no cavitacionales. Tanto para hombres como para mujeres los indicadores fueron más altos en el nivel educativo de la secundaria y se presentaron diferencias significativas $(\mathrm{p}<0,05)$, excepto para cariados no cavitacionales y cariados en conjunto.

La prevalencia de caries dental en ambos sexos, incluyendo lesiones no cavitacionales, es del 39\%. Tanto la prevalencia como la experiencia con caries crecen conforme aumenta la edad en hombres y en mujeres. Estos dos indicadores tienen mayor frecuencia en el nivel educativo de la secundaria, con excepción de la prevalencia de caries dental cuando se incluyen las lesiones no cavitacionales en las mujeres, que es mayor en la población con estudios primarios (tabla 2).

Al observar el promedio cop en el tercio más afectado de la población (sic, tabla 3), la media en hombres es de 2,70 cuando se calcula a partir del $\operatorname{CoP}_{\mathrm{T}}$ y del 4,36 para el caso del $\mathrm{COP}_{\mathrm{M}}$. Estos indicadores son menores en mujeres ( 2,56 y 3,36 , respectivamente). Se observan las mismas tendencias en la presentación de estos indicadores: el sIC aumenta con la edad y es mayor en personas de secundaria. 
Tabla 1. Índice cop y sus componentes según diferentes variables. Área rural del municipio de Jericó (Antioquia), 2013

\begin{tabular}{|c|c|c|c|c|c|c|c|c|c|c|c|c|c|c|c|}
\hline \multirow{2}{*}{ Variables } & \multirow{2}{*}{$\mathbf{N}$} & \multicolumn{13}{|c|}{ Promedio (+-DS) } & \multirow[b]{2}{*}{$p^{*}$} \\
\hline & & $\mathrm{C} 1$ & $p^{*}$ & $\mathrm{C} 2$ & $p^{\star}$ & $\mathrm{C} 1+\mathrm{C} 2$ & $p^{*}$ & $\mathbf{O}$ & $p^{*}$ & $\mathbf{P}$ & $p^{*}$ & $\mathrm{COP}_{\mathrm{T}}$ & $p^{*}$ & $\mathrm{COP}_{\mathrm{M}}$ & \\
\hline \multicolumn{16}{|c|}{ Hombres } \\
\hline \multicolumn{16}{|l|}{ Edad } \\
\hline 6 a 9 & 102 & $0,37(1,30)$ & \multirow{3}{*}{0,287} & $0,23(0,61)$ & \multirow{3}{*}{$<0,001$} & $0,60(1,67)$ & \multirow{3}{*}{$<0,001$} & $0,03(0,17)$ & \multirow{3}{*}{$<0,001$} & $0,00(0,00)$ & \multirow{3}{*}{$<0,001$} & $0,25(0,62)$ & \multirow{3}{*}{$<0,001$} & $0,63(1,67)$ & \multirow{3}{*}{$<0,001$} \\
\hline 10 a 12 & 81 & $0,68(1,73)$ & & $0,63(1,03)$ & & $1,31(2,05)$ & & $0,22(0,63)$ & & $0,01(0,11)$ & & $0,86(1,31)$ & & $1,54(2,15)$ & \\
\hline 13 y más & 86 & $0,94(2,23)$ & & $0,90(1,73)$ & & $1,84(3,00)$ & & $0,69(1,30)$ & & $0,24(0,67)$ & & $1,83(2,48)$ & & $2,77(3,48)$ & \\
\hline \multicolumn{16}{|c|}{ Nivel de estudios } \\
\hline Primaria & 178 & $0,62(1,73)$ & \multirow{2}{*}{0,765} & $0,53(1,26)$ & \multirow{2}{*}{0,532} & $1,15(2,38)$ & \multirow{2}{*}{0,578} & $0,12(0,47)$ & \multirow{2}{*}{$<0,001$} & $0,01(0,11)$ & \multirow{2}{*}{$<0,001$} & $0,66(1,40)$ & \multirow{2}{*}{$<0,001$} & $1,28(2,43)$ & \multirow{2}{*}{0,008} \\
\hline Secundaria & 91 & $0,69(1,87)$ & & $0,63(1.12)$ & & $1,32(2,22)$ & & $0,64(1,27)$ & & $0,23(0,65)$ & & $1,49(2,17)$ & & $2,19(2,98)$ & \\
\hline $\begin{array}{l}\text { Total } \\
\text { hombres }\end{array}$ & 269 & $0,65(1,78)$ & & $0,56(1,22)$ & & $1,21(2,33)$ & & $0,30(0,86)$ & & $0,08(0,40)$ & & $0,94(1,74)$ & & $1,59(2,66)$ & \\
\hline \multicolumn{16}{|c|}{ Mujeres } \\
\hline \multicolumn{16}{|l|}{ Edad } \\
\hline 6 a 9 & 99 & $0,19(0,65)$ & \multirow{3}{*}{0,368} & $0,27(0,64)$ & \multirow{3}{*}{$<0,001$} & $0,46(0,91)$ & \multirow{3}{*}{0,002} & $0,10(0,39)$ & \multirow{3}{*}{$<0,001$} & $0,00(0,00)$ & \multirow{3}{*}{0,004} & $0,37(0,83)$ & \multirow{3}{*}{$<0,001$} & $0,57(1,03)$ & \multirow{3}{*}{$<0,001$} \\
\hline 10 a 12 & 78 & $0,49(1,27)$ & & $0,46(0,86)$ & & $0,15(1,71)$ & & $0,13(0,54)$ & & $0,06(0,47)$ & & $0,65(1,15)$ & & $1,14(1,93)$ & \\
\hline 13 y más & 88 & $0,40(1,33)$ & & $1,03(1,53)$ & & $1,43(2,03)$ & & $0,55(1,12)$ & & $0,13(0,50)$ & & $1,70(1,93)$ & & $2,10(2,28)$ & \\
\hline \multicolumn{16}{|c|}{ Nivel de estudios } \\
\hline Primaria & 163 & $0,43(1,22)$ & & $0,45(0,85)$ & & $0,88(1,62)$ & & $0,11(0,47)$ & & $0,01(0,11)$ & & $0,58(1,02)$ & & $1,01(1,74)$ & \\
\hline Secundaria & 102 & $0,22(0,89)$ & 0,120 & $0,78(1,43)$ & 0,010 & $1,00(1,67)$ & 0,417 & $0,49(1,06)$ & $<0,001$ & $0,14(0,60)$ & 0,010 & $1,41(1,91)$ & $<, 001$ & $1,63(2,08)$ & 0,003 \\
\hline $\begin{array}{l}\text { Total } \\
\text { mujeres }\end{array}$ & 265 & $0,35(1,11)$ & & $0,58(1,18)$ & & $0,93(1,64)$ & & $0,26(0,78)$ & & $0,06(0,39)$ & & $0,90(1,48)$ & & $1,25(1,90)$ & \\
\hline
\end{tabular}

N: tamaño de la muestra; DS: desviación estándar; $\mathrm{C} 1$ : cariados no cavitacionales; C2: cariados cavitacionales; O: obturados; P: perdidos; $\operatorname{COPD}_{\mathrm{T}}$ : índice COP tradicional; $\operatorname{COPD}_{\mathrm{M}}$ : índice COP modificado.

$p^{*}$ : Pruebas no paramétricas U de Mann Whitney (nivel de estudios) y Kruskal Wallis (edad).

Fuente: elaboración propia

Tabla 2. Prevalencia y experiencia con caries según diferentes variables. Área rural del municipio de Jericó (Antioquia), 2013

\begin{tabular}{|c|c|c|c|c|c|c|c|c|c|c|c|c|c|c|c|c|c|}
\hline \multirow{3}{*}{ Variables } & \multicolumn{4}{|c|}{ Prevalencia de caries } & \multicolumn{4}{|c|}{$\begin{array}{l}\text { Experiencia } \\
\text { con caries }\end{array}$} & \multirow{3}{*}{ Variables } & \multicolumn{4}{|c|}{ Prevalencia de caries } & \multicolumn{4}{|c|}{$\begin{array}{l}\text { Experiencia } \\
\text { con caries }\end{array}$} \\
\hline & \multicolumn{2}{|c|}{$\mathrm{C} 2>0$} & \multicolumn{2}{|c|}{$\mathrm{C} 1+2>0$} & \multicolumn{2}{|c|}{$\mathrm{COP}_{\mathrm{T}}>0$} & \multicolumn{2}{|c|}{$\mathrm{COP}_{M}>0$} & & \multicolumn{2}{|c|}{$\mathrm{C} 2>0$} & \multicolumn{2}{|c|}{$\mathrm{C} 1+2>0$} & \multicolumn{2}{|c|}{$\mathrm{COP}_{\mathrm{T}}>0$} & \multicolumn{2}{|c|}{$\mathrm{COP}_{M}>0$} \\
\hline & $\mathrm{n}$ & $\%$ & $\mathbf{n}$ & $\%$ & n & $\%$ & $\mathrm{n}$ & $\%$ & & $\mathbf{n}$ & $\%$ & $\mathbf{n}$ & $\%$ & $\mathbf{n}$ & $\%$ & $\mathbf{n}$ & $\%$ \\
\hline \multicolumn{9}{|c|}{ Hombres } & \multicolumn{9}{|c|}{ Mujeres } \\
\hline \multicolumn{9}{|l|}{ Edad } & \multicolumn{9}{|l|}{ Edad } \\
\hline 6 a 9 & 15 & 14,7 & 24 & 23,5 & 18 & 17,6 & 27 & 26,5 & 6 a 9 & 20 & 20,2 & 27 & 27,3 & 23 & 23,2 & 30 & 30,3 \\
\hline 10 a 12 & 30 & 37,0 & 42 & 51,9 & 34 & 42,0 & 45 & 55,6 & 10 a 12 & 23 & 29,5 & 31 & 39,7 & 28 & 35,9 & 35 & 44,9 \\
\hline 13 y más & 31 & 36,0 & 40 & 46,5 & 48 & 55,8 & 53 & 61,6 & 13 y más & 38 & 43,2 & 44 & 50,0 & 53 & 60,2 & 57 & 64,8 \\
\hline \multicolumn{9}{|c|}{ Nivel de estudios } & \multicolumn{9}{|c|}{ Nivel de estudios } \\
\hline Primaria & 48 & 27,0 & 68 & 38,2 & 56 & 31,5 & 75 & 42,1 & Primaria & 48 & 29,4 & 63 & 38,7 & 54 & 33,1 & 68 & 41,7 \\
\hline Secundaria & 28 & 30,8 & 38 & 41,8 & 44 & 48,4 & 50 & 54,9 & Secundaria & 33 & 32,4 & 39 & 38,2 & 50 & 49,0 & 54 & 52,9 \\
\hline Total hombres & 76 & 28,3 & 106 & 39,4 & 100 & 37,2 & 125 & 46,5 & Total mujeres & 81 & 30,6 & 102 & 38,5 & 104 & 39,2 & 122 & 46,0 \\
\hline
\end{tabular}

C1: cariados no cavitacionales; C2: cariados cavitacionales; $\mathrm{COPD}_{\mathrm{T}}$ : índice $\mathrm{COP}$ tradicional; $\mathrm{COPD}_{\mathrm{M}}$ : índice $\mathrm{COP}$ modificado.

Fuente: elaboración propia 
Tabla 3. Índice de caries significativo (SIC)* según diferentes variables. Área rural del municipio de Jericó (Antioquia), 2013

\begin{tabular}{|c|c|c|}
\hline Variables & $\mathrm{SiC}_{1}$ & $\mathrm{SiC}_{2}$ \\
\hline \multicolumn{3}{|c|}{ Hombres } \\
\hline \multicolumn{3}{|l|}{ Edad } \\
\hline 6 a 9 & 0,76 & 1,88 \\
\hline 10 a 12 & 2,33 & 3,85 \\
\hline 13 y más & 4,48 & 6,79 \\
\hline \multicolumn{3}{|l|}{ Nivel de estudios } \\
\hline Primaria & 1,98 & 3,59 \\
\hline Secundaria & 3,93 & 5,63 \\
\hline Total hombres & 2,70 & 4,36 \\
\hline \multicolumn{3}{|c|}{ Mujeres } \\
\hline \multicolumn{3}{|l|}{ Edad } \\
\hline 6 a 9 & 1,12 & 1,70 \\
\hline 10 a 12 & 1,88 & 3,08 \\
\hline 13 y más & 4,03 & 4,83 \\
\hline \multicolumn{3}{|l|}{ Nivel de estudios } \\
\hline Primaria & 1,74 & 2,78 \\
\hline Secundaria & 3,74 & 4,15 \\
\hline Total mujeres & 2,52 & 3,36 \\
\hline
\end{tabular}

* SiC1: calculado sobre el Índice cop tradicional; SiC2: calculado sobre el Îndice cop modificado.

Nota: se calculó el promedio a partir de las fórmulas en hoja de cálculo disponibles en: http://www.mah.se/CAPP/Methods-and-Indices/forCaries-prevalence/Significant-Caries-Index/Online-Calculation-ofDMFTdmft-and-SIC-Index-I

Fuente: elaboración propia

En cuanto al IHos (tabla 4), el promedio es mucho menor en mujeres $(1,25$ IC $95 \% 1,18-1,32)$ y con diferencias estadísticamente significativas con respecto a los hombres $(\mathrm{p}<0,001)$. Existen disparidades de acuerdo con el nivel educativo y la edad, que son significativas en las mujeres, acorde con el nivel educativo. Por último, teniendo en cuenta los niveles de higiene oral (tabla 5), las mujeres presentan mayores frecuencias de niveles buenos con respecto a los hombres, y estos últimos revelaron la mayor frecuencia de niveles regular y malo, con diferencias estadísticamente significativas en la distribución de las frecuencias $(\mathrm{p}<0,001)$, igual que en los promedios del inos; se presentaron diferencias en las mujeres de acuerdo con el nivel educativo.
Tabla 4. Índice de Higiene Oral Simplificado según diferentes variables. Área rural del municipio de Jericó (Antioquia), 2013

\begin{tabular}{|c|c|c|c|c|c|}
\hline \multirow{2}{*}{ Variables } & \multicolumn{5}{|c|}{ IHOS } \\
\hline & Promedio & IC $_{95 \%}$ & Mediana & RIC & $p^{*}$ \\
\hline \multicolumn{6}{|c|}{ Hombres } \\
\hline \multicolumn{6}{|l|}{ Edad } \\
\hline 6 a 9 & 1,43 & $1,31-1,53$ & 1,33 & $1,00-1,82$ & \multirow{3}{*}{0,303} \\
\hline 10 a 12 & 1,65 & $1,51-1,78$ & 1,50 & $1,16-2,16$ & \\
\hline 13 y más & 1,46 & $1,32-1,60$ & 1,32 & $0.98-1,82$ & \\
\hline \multicolumn{6}{|c|}{ Nivel de estudios } \\
\hline Primaria & 1,55 & $1,46-1,64$ & 1,50 & $1,16-1,98$ & \multirow{2}{*}{0,078} \\
\hline Secundaria & 1,41 & $1,28-1,54$ & 1,32 & $0,98-1,82$ & \\
\hline Total hombres & 1,50 & $1,43-1,57$ & 1,33 & $1,00-1,82$ & $<0,001$ \\
\hline \multicolumn{6}{|c|}{ Mujeres } \\
\hline \multicolumn{6}{|l|}{ Edad } \\
\hline 6 a 9 & 1,26 & $1,16-1,37$ & 1,16 & $0,82-1,50$ & \multirow{3}{*}{0,539} \\
\hline 10 a 12 & 1,27 & $1,13-1,42$ & 1,16 & $0,82-1,66$ & \\
\hline 13 y más & 1,21 & $1,08-1,34$ & 1,00 & $0,82-1,66$ & \\
\hline \multicolumn{6}{|c|}{ Nivel de estudios } \\
\hline Primaria & 1,36 & $1,27-1,46$ & 1,20 & $0,98-1,66$ & \multirow{2}{*}{0,006} \\
\hline Secundaria & 1,07 & $0,97-1,16$ & 1,00 & $0,66-1,32$ & \\
\hline Total mujeres & 1,25 & $1,18-1,32$ & 1,16 & $0,82-1,66$ & $<0,001$ \\
\hline
\end{tabular}

$p^{*}$ : Pruebas no paramétricas: Prueba de la Mediana. RIC: Rango Intercuartílico.

Fuente: elaboración propia

Tabla 5. Nivel de higiene oral según diferentes variables. Área rural del municipio de Jericó (Antioquia), 2013

\begin{tabular}{|c|c|c|c|c|}
\hline \multirow{2}{*}{ Variables } & \multicolumn{4}{|c|}{ Nivel de Higiene Oral } \\
\hline & Bueno & Regular & Malo & $p^{*}$ \\
\hline \multicolumn{5}{|c|}{ Hombres } \\
\hline \multicolumn{5}{|l|}{ Edad } \\
\hline 6 a 9 & 44,1 & 54,9 & 1,0 & \multirow{3}{*}{0,240} \\
\hline 10 a 12 & 28,4 & 69,1 & 2,5 & \\
\hline 13 y más & 40,7 & 57,0 & 2,3 & \\
\hline \multicolumn{5}{|c|}{ Nivel de estudios } \\
\hline Primaria & 34,8 & 63,5 & 1,7 & \multirow{2}{*}{0,236} \\
\hline Secundaria & 45,1 & 52,7 & 2,2 & \\
\hline Total hombres & 38,3 & 59,9 & 1,9 & $<0,001$ \\
\hline \multicolumn{5}{|c|}{ Mujeres } \\
\hline \multicolumn{5}{|l|}{ Edad } \\
\hline 6 a 9 & 56,6 & 43,40 & 0,0 & \multirow{3}{*}{0,516} \\
\hline 10 a 12 & 53,8 & 44,9 & 1,3 & \\
\hline 13 y más & 61,4 & 38,6 & 0,0 & \\
\hline \multicolumn{5}{|c|}{ Nivel de estudios } \\
\hline Primaria & 51,5 & 47,9 & 0,6 & \multirow{2}{*}{0,04} \\
\hline Secundaria & 66,7 & 33,3 & 0,0 & \\
\hline Total mujeres & 57,4 & 42,3 & 1,9 & $<0,001$ \\
\hline
\end{tabular}

$p^{*}$ : Prueba Chi cuadrado para distribución de proporciones.

Fuente: elaboración propia 


\section{Discusión}

Los resultados de este estudio reflejaron que la prevalencia y la experiencia con caries aumentó con la edad tanto en hombres como en mujeres. Al evaluar los índices de caries se encontraron diferencias estadísticamente significativas en relación con el $\mathrm{COPD}_{\mathrm{T}}$ por edad y grado de escolaridad, tanto para hombres como para mujeres. En relación con el índice $\mathrm{COPD}_{\mathrm{M}}$, las diferencias sólo se evidenciaron por edades para ambos sexos. El índice significativo de caries (SIC) aumentó respecto a la edad y al grado de escolaridad, en ambos sexos. Por último, el índice de higiene bucal fue mejor en mujeres que en hombres; entre estos últimos fue mayor en las edades de 10 a 12 años, y en grados de escolaridad más altos.

Al comparar los resultados de los índices con el último Estudio Nacional de Salud Bucal III [6] se encontró que el $20 \%$ de los menores de siete años tuvo experiencia con caries; esto concuerda con los resultados de esta investigación, que muestra que en las edades de seis a nueve años la experiencia con caries afectó al $18 \%$ de los hombres y al $23 \%$ de las mujeres. Los resultados reflejaron, además, que al aumentar la edad se incrementó la experiencia con caries, tal y como se observa en el ENSAB III [6]; sin embargo, el incremento a nivel nacional en este estudio fue mucho más alto que en la población rural escolar de Jericó. Datos nacionales mostraron que el $72 \%$ de la población evaluada, a los 12 años tuvo experiencia con caries; en el grupo de 15 a 19 años fue del $90 \%$.

Pasando del contexto nacional al departamental, al comparar los resultados con el levantamiento de la línea base realizado en Antioquia en el 2011 [7], se encuentra que a los 12 años la experiencia con caries es del $62 \%$, mientras que en la población evaluada en Jericó, en el rango de edad que incluye a los 12 años, es más bajo ( $42 \%$ para hombres y $36 \%$ para mujeres). Al evaluar la experiencia con caries, incluyendo las lesiones ocasionadas por caries limitadas al esmalte dental, los valores se incrementaron respecto a la experiencia con caries cavitacional, en el 14\% para hombres y el $9 \%$ para mujeres, pero en el estudio departamental el incremento fue sólo del 3,5\%. En el último Monitoreo de Salud Bucal en escolares de instituciones oficiales de Medellín [16], la experiencia con caries a los 8 años fue del $18 \%$, a los 12 años del $34 \%$ y a los 15 años del $45 \%$. Dichos indicadores fueron más bajos que en la población escolar rural de Jericó. En otros municipios del departamento, por ejemplo, en Rionegro, se han identificado experiencias con caries del $72 \%$ de los hombres y del $68 \%$ de las mujeres en la edad de 12 años [17]; en Yondó, del $37 \%$ de los hombres y del $28,4 \%$ de las mujeres [18]; en Frontino, la experiencia, sin distinción por sexos, fue del 37\% [19]. En estos municipios la experiencia de caries es mucho más alta.

En Rionegro, la prevalencia de caries dental, incluyendo lesiones no cavitacionales, es del 71\% (hombres) y del 60\% (mujeres), teniendo en cuenta sólo los dientes con caries cavitacional [17]. Estos resultados muestran un comportamiento similar de la prevalencia en relación con la experiencia con caries, siendo comparativamente más alta en los hallazgos nacionales y departamentales que en Jericó; sin embargo, al compararlos con los de Medellín [16], los indicadores de Jericó son mucho mayores.

La valoración del índice $\operatorname{COPD}_{\mathrm{T}}$ en el ENSAB III [6], según los grupos de edades, mostró que a los siete años era de 0,3 (en la población escolar de Jericó fue de 0,25 en hombres de y 0,37 en mujeres). A los 12 años el co$\mathrm{PD}_{\mathrm{T}}$ fue de 2,3, mientras que en el presente estudio, en escolares incluidos en este rango de edad, fue mucho más bajo. En la población de 15 a 19 años fue de 5,2, mientras que en Jericó, en la población de 13 años fue también más bajo. En la línea de base departamental el $\mathrm{COPD}_{\mathrm{T}}$ fue de 1,9 para mujeres y 1,7 para hombres a la edad de 12 años, y el $\operatorname{COPD}_{\mathrm{M}}$ fue de 2,2 para mujeres y 2,0 para hombres [7], lo que muestra que son resultados más elevados que los de la población del presente estudio.

Los valores reportados por el sic fueron más bajos en comparación con los observados en la línea base del departamento de Antioquia, en los que a esta edad fue de 4,3 $\pm 1,8$ [7]. Sin embargo, en Medellín [16], a los 12 años fue de 2,1 $\pm 2,1$ a partir de $\operatorname{COPD}_{\mathrm{T}}$ y de $1,3 \pm 1,2$ tomado a partir del $\operatorname{COPD}_{\mathrm{M}}$, que son valores más bajos que los encontrados en Jericó. Otros estudios realizados en el departamento, que evaluaron el sic, reportan valores más altos en este grupo de edad, que incluyen 3,79 en hombres y 3,38 en mujeres [17]. La valoración de este índice aportó información valiosa sobre la polarización de la caries dental en un tercio de la población (tal y como lo calcula el sIC) [20], fenómeno evidenciado en grupos poblacionales con características sociales que los hace más vulnerables en múltiples aspectos y que además se ven reflejadas en los procesos de salud enfermedad, dando muestra de desigualdades sociales y en salubridad [21]. En Jericó, en esta población escolar rural este índice fue sensible y dio cuenta de que en un segmento importante de esta comunidad se duplicaron 
los promedios generales a partir de los índices $\operatorname{COPD}_{\mathrm{T}} \mathrm{y}$ $\mathrm{COPD}_{\mathrm{M}}$, indicando la necesidad de focalizar los esfuerzos en este grupo poblacional más afectado, en aras de disminuir las brechas sociales y económicas latentes.

En relación con la higiene bucal, en el último Estudio Nacional de Salud Bucal se reporta que el 82\% del total de las personas presentó placa al momento del examen, con mayor proporción en las personas menores de 20 años (95\%), hasta alcanzar menores proporciones en personas de edad avanzada (27\%), debido básicamente a la pérdida dentaria [6]. En el caso de Jericó, una importante proporción de hombres y mujeres presentaron índices de higiene bucal regulares o malos, de acuerdo con el inos (mayores a los reportados en el ENSAB III). Aunque los resultados deben interpretarse con cautela, teniendo en cuenta la dificultad de comparar exactamente los grupos analizados en el estudio local con el nacional.

Se encontraron diferencias importantes en los indicadores por sexo, por edad y por nivel educativo; en esto intervienen factores relacionados con las prácticas de autocuidado; la literatura ha demostrado que las mujeres presentan mejores prácticas de autocuidado y son las que utilizan con mayor frecuencia los servicios de salud y de salud bucal [22]. Esto mismo podría estar relacionado con la edad y el nivel educativo, en cuanto a la capacidad de mayor entendimiento del componente bucal, del proceso salud-enfermedad y mayores prácticas de autocuidado, ya que en la adolescencia la estética se puede considerar un factor importante, porque las relaciones sociales aumentan en este periodo de edad, lo cual puede ser decisivo en la adopción de prácticas saludables de salud bucal [23].

Como fortalezas que presenta este estudio puede decirse que contó con una amplia muestra de población escolar de primaria y secundaria de la zona rural de Jericó, lo cual permitió conocer el contexto global de la situación de caries dental e higiene bucal de la población de estudio. El análisis segmentado por sexo permitió la incorporación de la perspectiva de género en la investigación en salud, reconociendo que los determinantes no se distribuyen de igual manera entre hombres y mujeres [24, 25]. Uno de los investigadores participó activamente en el proceso de recolección de información, lo cual garantizó la calidad de las variables que se utilizaron.

Es importante mencionar que este estudio es previo a una fase de intervención que se realizará posteriormente en el municipio, por lo que la disponibilidad de las variables está supeditada a la información que se recolectó en las historias clínicas. Tampoco se tuvo información de la zona urbana, lo cual en posteriores estudios enriquecerá el análisis. Nuevos estudios deberían incorporar a otros grupos de edad e incluir otras variables relacionadas con estilos de vida, aspectos culturales y sociales de la familia y el entorno que permiten una visión más integral desde la óptica de los determinantes sociales de la salud.

\section{Conclusiones}

Los resultados de este estudio permiten concluir que la mayoría de los indicadores calculados se encontraron por debajo de los valores del ENSAB III, la línea de base en caries dental, Antioquia (Colombia), 2011, y algunos municipios del departamento. Sin embargo, se encuentran diferencias importantes según factores sociodemográficos y de un subgrupo de la población analizada que presenta peores indicadores, lo cual guarda coherencia con el fenómeno de la polarización de la caries dental. Es por ello que la problemática derivada del proceso de la caries dental en Jericó aún no está resuelta y falta focalizar esfuerzos en esta población rural, profundizando en investigar las necesidades sociales, culturales, económicas, demográficas y de acceso a los servicios de salud que podría presentar la población.

Es importante, además, continuar con un seguimiento y vigilancia a los indicadores, incluyendo además el estudio de otros factores determinantes del proceso salud-enfermedad que permitan un seguimiento más completo. Se deben incrementar los conocimientos, en especial en torno a los hábitos y prácticas de salud bucal, partiendo de las representaciones sociales de esta población, por medio de programas educativos adecuadamente orientados y planteados que hagan partícipes a las diferentes instituciones escolares, tanto urbanas como rurales, y por último se propone realizar un seguimiento y una evaluación constantes de las políticas y estrategias de salud bucal en Jericó, desde una perspectiva de los determinantes sociales de la salud, de una forma holística e integradora.

\section{Agradecimientos}

Al municipio de Jericó (Antioquia) y a la ese Hospital San Rafael y su gerente, Hernán Darío Aguilar Ramírez, por impulsar y permitir el desarrollo del presente 
estudio, en aras de mayor conocimiento de las condiciones de salud, en su componente bucal, en esta población.

\section{Referencias}

[1] Suárez-Zúñiga E, Velosa-Porras J. Comportamiento epidemiológico de la caries dental en Colombia. Univ Odontol. 2013; 32(68): 117-24.

[2] Escobar PG, Ortiz AC, Mejía LM. Caries dental en los menores de veinte años en Colombia: un problema de salud pública. Rev Fac Nac Salud Pública. 2009; 21(2): 107-18.

[3] Naranjo CD, Díaz ÍIM. La representación de la epidemiología de la caries en el mundo a través de mapas. Univ Odontol. 2012; 33(66): 41-50.

[4] Mejía R. Morbilidad oral. Investigación nacional de morbilidad. Estudio de recursos humanos para la salud y la educación médica en Colombia. Bogotá: Ministerio de Salud, Ascofame; 1971.

[5] Colombia. Ministerio de Salud. Instituto Nacional de Salud-ASCOFAME. Morbilidad oral. II Estudio Nacional. Bogotá: El Ministerio, Instituto Nacional de Salud; 1980.

[6] Colombia. Ministerio de Salud. III Estudio Nacional de Salud Bucal EnSAB III, 1998. Bogotá: El Ministerio; 1998.

[7] Ramírez-Puerta BS, Viñas-Sarmiento Y, López-Camacho V, Morales-Flórez JL. Línea de base en caries dental, Antioquia (Colombia), 2011. Rev Nac Odont. 2012; 8(15): 9-20.

[8] Vilchis DBC, Castillo REP, Clavel JFG. El concepto de caries: hacia un tratamiento no invasivo. Revista ADM. 2013; 70(2): 54-60.

[9] Piovano S, Squassi A, Bordoni N. Estado del arte de indicadores para la medición de caries dental. Rev Fac Odont uBA. 2010; 25(58): 29-43.

[10] Greene JC, Vermillion JR. The simplified oral hygiene index. J Am Dent Assoc. 1964; 68: 7-13.

[11] Klein H, Palmer C, Knutson J. Studies on dental caries. I, dental status and dental needs of elementary school. Pub Health Rep. 1938; 53: 751-65.

[12] Pitts N, Deery C, Fyffe H, Nugent Z. Caries prevalence surveys a multi-country comparison of caries diagnostic criteria. Community Dent health. 2000; 17: 196-203.

[13] Bratthall D. Introducing the significant caries index together with a proposal for a new global oral health goal for 12-year-olds. Internat Dent J. 2000; 50(6): 378-84.
[14] World Medical Association General Assembly. World Medical Association Declaration of Helsinki: ethical principles for medical research involving human subjects. J Intern Bioet. 2004; 15(1): 124-9.

[15] Colombia. Ministerio de Salud. Resolución nº 008430 de 1993 (4 de octubre de 1993). Por la cual se establecen las normas científicas, técnicas y administrativas para la investigación en salud. Bogotá: El Ministerio; 1993.

[16] Franco-Cortés A, Ramírez-Puerta B, Díaz-Mosquera P, Ochoa-Acosta E. VII monitoreo: caries dental y acceso a los servicios odontológicos en escolares de Medellín, 2009. Rev Salud Publica Med. 2010; 4(2): 71-86.

[17] Vivares-Builes A, Saldarriaga-Saldarriaga AF, MuñozPino N, Miranda-Galvis M, Colorado-Colorado K, Montoya-Zuluaga YP, et al. Caries dental y necesidades de tratamiento en los escolares de 12 años de las instituciones oficiales del municipio de Rionegro (Antioquia, Colombia), 2010. Rev Fac Odontol Univ Antioq. 2012; 23(2): 292-305.

[18] Martínez-Flórez LM, Marulanda-Montoya E, Noreña-Salazar MA, Agudelo-Suárez AA. Prevalencia de fluorosis y experiencia de caries dental en un grupo de escolares en el área urbana del municipio de Yondó (Antioquia, Colombia), 2010. Rev ces Odontol. 2011; 24(1): 9-16.

[19] Ramírez-Puerta BS, Franco-Cortés ÁM. Caries en dentición permanente en escolares de 8 a 12 y 15 años. $\mathrm{Mu}$ nicipio de Frontino, Colombia. Rev Nac Odontl. 2010; 6(11): 9-15.

[20] Macek MD, Heller KE, Selwltz RH, Manz MC. Is 75 percent of dental caries really found in 25 percent of the population? J Public Health Dent. 2004; 64(1): 20-5.

[21] Narvai PC, Frazão P, Roncalli AG, Antunes JLF, Camargos P, Ribeiro Y. Cárie dentária no Brasil: declínio, polarização, iniqüidade e exclusão social. Rev Panam Salud Publica. 2006; 19(6): 385-93.

[22] Fukai K, Takaesu Y, Maki Y. Gender differences in oral health behavior and general health habits in an adult population. Bull Tokyo Dent Col. 1999; 40(4): 187-93.

[23] Ochoa-Acosta EM, Roldán-Vargas O, Ramírez-Puerta BS, Franco-Cortés ÁM. Significados del autocuidado bucal para los escolares de instituciones educativas oficiales de Medellín. Rev Nac Odont. 2011; 7(11): 40-8.

[24] Kunkel SR, Atchley RC. Why gender matters: being female is not the same as not being male. An J Prev Med. 1996; 12(5): 294-6.

[25] Martinez-Mier EA, Zandona AF. The impact of gender on caries prevalence and risk assessment. Den Clin Nort Am. 2013; 57(2): 301-15. 